Received: 11.05 .2018

Revised: 30.05 .2018

Accepted: 15.06 .2018

DOI: $10.17804 / 2410-9908.2018 .3 .014-023$

\title{
COMPUTER-AIDED INTERSUBJECTIVE ASSIGNMENT OF OVERLAPS FOR SHAFT-TYPE FORGINGS
}

\author{
O. Yr. Muizemnek ${ }^{\text {a) }}$, A. V. Konovalov ${ }^{\text {b) }}$ and S. I. Kanyukov ${ }^{\text {() }}$ \\ Institute of Engineering Science, Ural Branch of the Russian Academy of Sciences, \\ 34 Komsomolskaya St., Ekaterinburg, Russian Federation \\ a) (iD https://orcid.org/0000-0002-8359-2579, هolga@imach.uran.ru; ${ }^{b}$ (iD https://orcid.org/ 0000-0001-9131-8636, \\ 凶avk@imach.uran.ru; ${ }^{\mathrm{c})}$ (D) https://orcid.org/ 0000-0001-5062-8080, @ t196at66@mail.ru \\ *Corresponding author. E-mail: olga@imach.uran.ru \\ Address for correspondence: 34, ul. Komsomolskaya, Ekaterinburg, Russian Federation \\ Tel.: +7 (343) 36230 26; fax: 3745330
}

The paper exemplifies the mechanism of choosing an intersubjective (rational) decision from a set of acceptable decisions by computer-aided assignment of overlaps for shaft-type forgings. Goals are formulated and formalized; membership functions of possible decisions corresponding to the set goals are defined. A criterion for the selection of a rational variant of overlap assignment from a set of acceptable decisions is developed.

Keywords: shaft, forging, computer simulation, formalized goal, membership function, intersubjective decision.

\section{References}

1. Karayel D., Ozkan S.S. Distributed multi-agent system approach for sheet metal forming. Journal of Materials Processing Technology, 2006, vol. 177, nos. 1-3, pp. 327-330. DOI: $10.1016 /$ j.jmatprotec.2006.04.039.

2. Zhang J., Chen Q. Characteristic finite strip analysis of sheet materials on multi-intelligence agents. Advanced Materials Research, 2013, vol. 662, pp. 657-660. DOI: 10.4028/www.scientific.net/ AMR.662.657.

3. Kaverin A. ADEM as State-of-the-Art Forging Process Planning. SAPR i Grafika, 2005, no. 3, p. 1. (In Russian).

4. Chesnokov V.S., Kaplunov B.G. Vozmishchev N.E. et al. Development and application of computer-aided design and modeling of hot forging processes. Kuznechno-Shtampovochnoe Proizvodstvo. Obrabotka Materialov Davleniem, 2008, no. 9, pp. 36-44. (In Russian).

5. Sakamoto S., Katayama T., Yokogawa R. et al. Construction of PC-based intelligent CAD system for cold forging process design - integration of CAD system and development of input method. Journal of Materials Processing Technology, 2001, vol. 119, nos. 1-3, pp. 58-64.

6. Jolgaf M., Hamouda A.M.S., Sulaiman S. et al. Development of a CAD/CAM system for the closed-die forging process. Journal of Materials Processing Technology, 2003, vol. 138, nos. 1-3, pp. 436-442. DOI 10.1108/02644400910943635.

7. Yang D.Y., Im Y.T., Yoo Y.C. et al. Development of integrated and intelligent design and analysis system for forging processes. CIRP Annals - Manufacturing Technology, 2000, vol. 49, no. 1 , pp. $177-180$.

8. Trubin V.N., Makarov V.I., Orlov S.N. et al. Sistema upravleniya kachestvom proektirovaniya tekhnologicheskikh protsessov kovki [Quality Management System of Forging Process Design]. Moscow, Mashinostroenie Publ., 1984, 184 p. (In Russian). 
9. Kanyukov S.I., Arzamastsev S.V. Computer-aided design system for the process of forging of stepped shafts. Kuznechno-Shtampovochnoe Proizvodstvo. Obrabotka Materialov Davleniem, 1995, no. 9, pp. 13-14. (In Russian).

10. Konovalov A.V., Arzamastsev S.V., Shalyagin S.D. et al. Intelligent computer-aided system of shaft forging on hammers. Zagotovitelnye Proizvodstva v Mashinostroenii, 2010, no. 1, pp. 20-23. (In Russian).

11. Konovalov A.V., Gagarin P.Yu., Shalyagin S.D. An object model of forging short blanks. Avtomatizatsiya i Sovremennye Tekhnologii, 2010, no. 6, pp. 11-15. (In Russian).

12. GOST 7062-90. Carbon and alloyed steel forgings fabricated by press forging. Allowances and tolerances. (In Russian).

13. Luger D.F. Iskusstvennyi intellekt: strategii i metody resheniya slozhnykh problem [Artificial Intelligence: Strategies and Methods for Solving Complex Problems, 4th ed.]. M., Izdatelskiy Dom Williams Publ., 2003, 864 p. (In Russian).

14. Stuart R., Norvig P. Iskusstvennyi intellekt: sovremennyi podkhod [Artificial Intelligence: Modern Approach, 2 ed.]. M., Izdatelskiy Dom Williams Publ., 2006. 1408 p. (In Russian).

15. Borgest N.M. Ontologiya proektirovaniya: teoreticheskie osnovy. Ch. 1. Ponyatiya $i$ printsipy: Uchebnoe posobie [Ontology of Design: Theoretical Foundations. Part 1. Concepts and Principles]. Samara, Izd-vo SGAU Publ., 2010, 92 p. (In Russian).

16. Vittikh V.A. Vvedenie v teoriyu intersubyektivnogo upravleniya [Introduction to the Intersubjective Control Theory]. Samara: Samarskiy Nauchnyi Centr RAN, 2013, 60 p. (In Russian).

17. Ryzhov A.P. Elementy teorii nechetkikh mnozhestv $i$ ee prilozheniy [Elements of the Fuzzy Set Theory and Its Applications]. M., Dialog-MGU Publ., 1998, 81 p. (In Russian).

18. Bellman R., Zade L. Prinyatie resheniy $v$ rasplyvchatykh usloviyakh. Voprosy analiza $i$ protsedury prinyatiya resheniy [Decision-Making under Fuzzy Conditions. Analysis and DecisionMaking Procedures]. M., Mir Publ., 1976, pp. 172-215. (In Russian). 
Подана в журнал: 11.05 .2018

УДК 004.89; 004.827; 621.73

DOI: $10.17804 / 2410-9908.2018 .3 .014-023$

\title{
АВТОМАТИЗИРОВАННОЕ ИНТЕРСУБЪЕКТИВНОЕ НАЗНАЧЕНИЕ НАПУСКОВ НА ПОКОВКИ ТИПА ВАЛОВ
}

\author{
О. Ю. Муйземнек ${ }^{\text {a)* }}$, А. В. Коновалов ${ }^{\text {б) }}$, С. И. Канюков ${ }^{\text {в) }}$ \\ Федеральное государственное бюджетное учреждение науки \\ Институт машиноведения Уральского отделения Российской академии наук, \\ ул. Комсомольская, 34, Екатеринбург, Российская Федерация
}

a) iD https://orcid.org/0000-0002-8359-2579, هolga@imach.uran.ru; ${ }^{6)}$ iD https://orcid.org/ 0000-0001-9131-8636, 凶avk@imach.uran.ru; ${ }^{\text {B) }}$ iD https://orcid.org/ 0000-0001-5062-8080, \196at66@mail.ru

\author{
*Ответственный автор. Электронная почта: olga@imach.uran.ru \\ Адрес для переписки: ул. Комсомольская, 34, Екатеринбург, Российская Федерация. \\ Тел.: +7 (343) 362-30-26; факс: 374-53-30
}

В статье описывается механизм выбора интерсубъективного (рационального) решения из множества допустимых решений, на примере решения задачи автоматизированного назначения напусков на поковки типа валов. Сформулированы и формализованы цели, определены функции принадлежности возможных решений поставленным целям и разработан критерий выбора рационального варианта назначения напусков из множества допустимых вариантов.

Ключевые слова: вал, поковка, компьютерное моделирование, формализованная цель, функиия принадлежности, интерсубъективное решение.

\section{1. Введение}

Технологическая подготовка кузнечного производства включает в себя проектирование поковки и разработку технологического процесса ковки и связана с необходимостью выполнения многочисленных расчетов, подготовкой и оформлением большого объема технической документации, необходимой для запуска изделий в производство. Эффективным средством сокращения трудоемкости и сроков технологической подготовки и повышением качества проектирования является автоматизированное проектирование технологических процессов с помощью систем автоматизированного проектирования (САПР). В качестве примеров таких разработок в рассматриваемой предметной области в нашей стране и за рубежом можно привести работы $[1,2]$ в области листовой штамповки, работы [3-7] - в области холодной и горячей штамповки и работы [8-11] в области свободной ковки. В Институте машиноведения УрО РАН (г. Екатеринбург) разработана интеллектуальная система автоматизированного проектирования технологии ковки валов на прессах (САПР «ТЕХНОЛОГ»).

Начальным этапом проектирования технологии ковки является проектирование поковки, т. е. назначение на исходную деталь припусков и кузнечных напусков. Назначение припусков производится путем добавления слоя металла на поверхность детали, подлежащего удалению при последующей механической обработке с целью ликвидации дефектов ковки и термообработки, осуществляется в соответствии с рекомендациями ГОСТ 7062-90 [12] и обычно затруднений не вызывает.

Решение задачи назначения напусков на поковку далеко не однозначно, поскольку регламентирующие правила ГОСТ 7062-90 и технологических инструкций предприятий в основном оговаривают ситуации, которые допускать нельзя. Следовательно, результатом решения в общем случае является множество допустимых решений, каждое из которых в 
принципе позволит получить годную поковку для выбра наиболее рационального решения в зависимости от поставленных целей.

Как правило, разработчики систем автоматизированного проектирования технологических процессов (САПР ТП) ковки при назначении напусков на поковку преследуют цель экономию металла, т.е. пытаются назначать минимально возможные напуски. Однако такой подход в общем случае нельзя считать единственно правильным. Другой целью является снижение трудоемкости изготовления поковки. Дополнительные напуски упрощают контур поковки, что приводит к уменьшению времени ее ковки. Может оказаться, что экономия, достигнутая при упрощении технологического процесса изготовления поковки, перевесит стоимость потерь металла, возникших из-за излишне назначенных напусков.

Цель настоящей работы - разработка механизма автоматизированного управления процессом назначения напусков на поковки типа валов с учетом разных поставленных целей.

Современные САПР ТП ковки представляют собой диалоговые автоматизированные системы с большой степенью интерактивности, при работе с которыми пользователь имеет возможность корректировать автоматически спроектированную поковку. Однако естественным стремлением разработчиков САПР всегда было и остается сокращение доли участия человека в процессе автоматизированного проектирования путем разработки более совершенных алгоритмов и программ, чему способствовало постоянное развитие теории искусственного интеллекта: объектно-ориентированный, мультиагентный, гибридный подходы [13], генетическое программирование [14], онтология проектирования [15], механизмы теории интерсубъективного управления [16].

Рассмотрим этот механизм на примере конкретной детали «Промвал». Деталь с назначенными припусками будем называть базовой. На рис. 1 приведен пример эскиза базовой детали «Промвал» (в скобках указаны размеры чистовой детали) и несколько возможных вариантов назначения напусков.

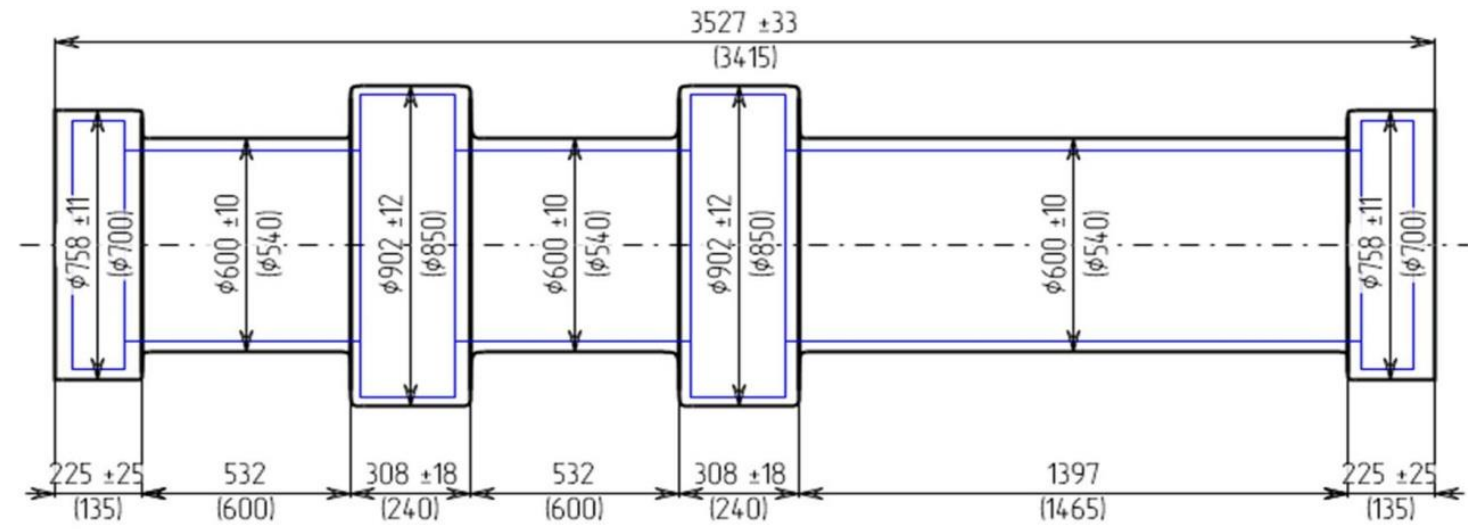

Вариант 1

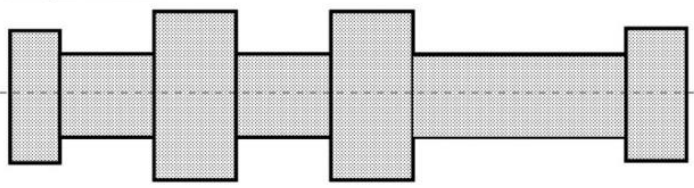

Вариант 3

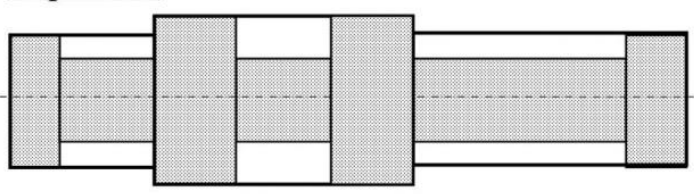

Вариант 2

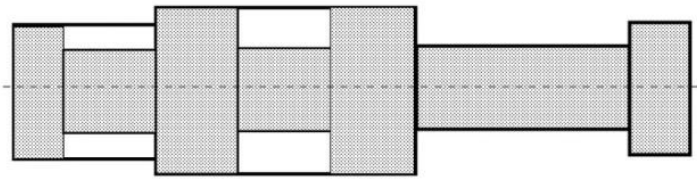

Вариант 4

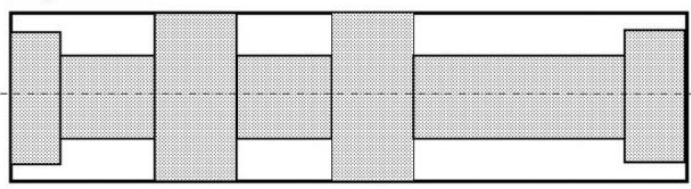

Рис. 1. Эскиз базовой детали «Промвал» и варианты назначения напусков 
Естественно, каждое решение задачи на рис. 1 следует считать субъективным, и вопрос состоит в том, какое из предложенных допустимых субъективных решений является наиболее рациональным (интерсубъективным) в зависимости от целей, которые при этом преследуются.

Для упрощения дальнейших рассуждений из четырех вариантов назначения напусков на рассматриваемую базовую деталь более подробно рассмотрим два первых варианта, спроектированных САПР «ТЕХНОЛОГ» и изображенных на рис. 2. Отметим, что оба указанных на этом рисунке варианта удовлетворяют требованиям ГОСТ 7062-90 и, следовательно, являются допустимыми.

В общем случае для решения задачи выбора нужного решения из множества возможных необходимо прежде всего сформулировать цели, которых хотелось бы достичь. Например, Цель 1 - экономия металла и Цель 2 - снижение трудоемкости изготовления поковки. Понятно, что эти цели являются противоречивыми и их приоритеты (важности) зависят прежде всего от объема заказа, стоимости металла, производственных условий предприятия и т. д.

Очевидно, что решение задачи назначения напусков на базовую деталь в общем случае должно учитывать обе цели в зависимости от их важностей. В теории интерсубъективного управления [16] такие решения, получаемые на основе «взаимопонимания» и консенсуса противоречивых целей, называют интерсубъективными. Для формализации процесса получения интерсубъективных решений удобно использовать теорию нечетких множеств [17] и теорию принятия решений в расплывчатых условиях [18], которые позволяют создать аппарат моделирования человеческих рассуждений и оценивать эти рассуждения при принятии решений, что естественно повышает интеллектуальный уровень САПР.

Прежде всего, необходимо формализовать цели, которые хотелось бы достичь.

Вариант 1

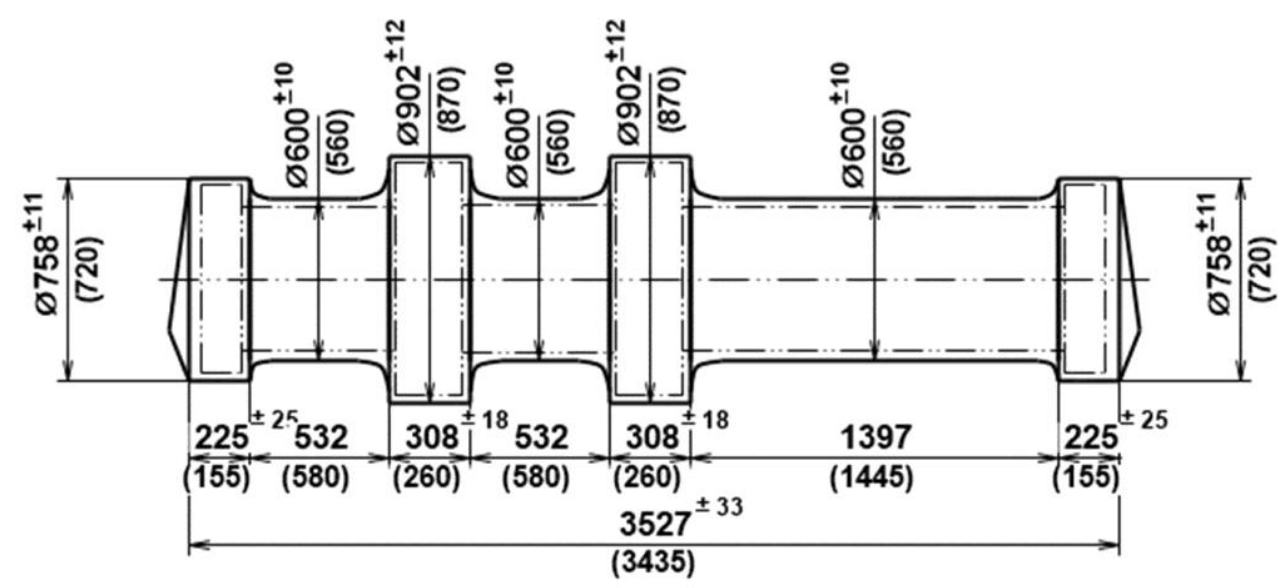

Вариант 2

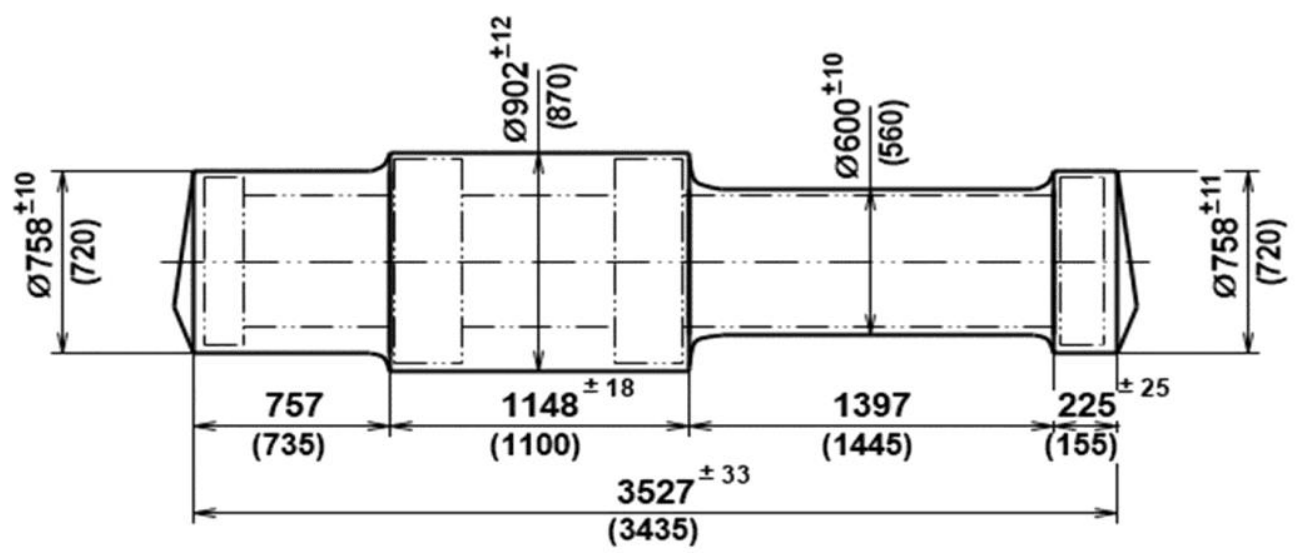

Рис. 2. Два варианта назначения напусков на базовую деталь

Muizemnek O. Yu., Konovalov A. V., Kanyukov S. I. Computer-aided intersubjective assignment of overlaps for shaft-type forgings // Diagnostics, Resource and Mechanics of materials and structures. - 2018. - Iss. 3. - P. 14-23. - 
Цель 1 - экономия металла. Достигается при минимальной массе спроектированной поковки. Степень достижения этой цели для каждого варианта спроектированной поковки, представленного на рис. 2, будем оценивать относительным расстоянием $r_{1}$ от этого варианта до базовой детали, которое будем вычислять по формуле:

$$
r_{1}=\frac{M-M_{B}}{M_{\Gamma}-M_{B}} \geq 0,
$$

где $M$ - масса допустимой поковки; $M_{5}=10550$ кг - масса базовой детали, представленной на рис. $1 ; M_{\Gamma}=17683$ кг - масса поковки на рис. 1 (вариант 4), которую назовем габаритной поковкой.

Отметим, что если $M=M_{5}$, то $r_{1}=0$, и допустимый вариант поковки совпадает с базовой деталью, т.е. находится от нее с точки зрения массы на минимальном расстоянии. При этом напуски на базовую деталь не назначаются.

Цель 2 - снижение трудоемкости изготовления поковки. Логично связать эту цель с объемом металла, который необходимо сместить, если бы исходной заготовкой для каждого варианта спроектированной поковки служила габаритная поковка (см. рис. 1, вариант 4).

Для формализации Цели 2 рассмотрим алгоритм расчета смещенного объема в случае преобразования цилиндрической ступени с размерами $D_{1}, L_{1}$ (стартовой ступени) в цилиндрическую ступень с размерами $D_{2}, L_{2}$ (конечную ступень).

Пусть $D, L$ - текущие размеры промежуточных ступеней между стартовой и конечной. Объем $V$ ступени в любой момент ее деформации есть величина постоянная. При этом

$$
V=\pi \frac{D_{1}^{2}}{4} L_{1}=\pi \frac{D_{2}^{2}}{4} L_{2}=\pi \frac{D^{2}}{4} L .
$$

Величина $L$ связана с $D$ соотношением

$$
L=\frac{4 V}{\pi D^{2}}
$$

Приращение смещенного объема $v^{C}$ ступени при бесконечно малом изменении ее диаметра $d D$ описывается выражением

$$
d v^{C}=\frac{\pi}{4}\left[D^{2}-(D-d D)^{2}\right] L .
$$
получим

После подстановки в выражение (2) формулы (1) и несложных преобразований

$$
d v^{C}=2 V \frac{d D}{D}, \quad v^{C}=\int_{D_{2}}^{D_{1}} d v^{C}=V \ln \left(\frac{D_{1}^{2}}{D_{2}^{2}}\right)
$$

Нетрудно убедиться, что когда цилиндрическая ступень с размерами $D_{1}, L_{1}$ преобразуется в коническую ступень с размерами $d_{2}, D_{2}, L_{2}$, смещенный объем $v^{c}$ вычисляется по формуле 


$$
v^{C}=V \ln \left(\frac{3 D_{1}^{2}}{d_{2}^{2}+d_{2} D_{2}+D_{2}^{2}}\right),
$$

где $d_{2}, D_{2}$ - диаметры конической ступени.

Смещенный объем $V^{C}$ для каждого варианта спроектированной поковки на рис. 2 определяется выражением

$$
V^{C}=\sum_{i=1}^{n} v_{i}^{C}
$$

где $v_{i}^{C}$ - смещенный объем $i$-й ступени поковки; $n$ - количество ступеней поковки.

Относительное расстояние $r_{2}$ от спроектированной поковки до габаритной с точки зрения Цели 2 определим как

$$
r_{2}=\frac{V^{C}}{V_{B}^{C}} \geq 0
$$

где $V_{E}^{C}$ - смещенный объем, рассчитанный для базовой детали. Очевидно, что если $V^{C}=0$, то $r_{2}=0$, т. е. смещения металла нет, и спроектированная поковка совпадает с габаритной.

Поскольку Цель 1 достигается при $r_{1}=0$, а Цель 2 - при $r_{2}=0$, то для выбора интерсубъективного варианта из допустимых вариантов, представленных на рис. 2, необходимо определить функцию принадлежности [17] каждого допустимого варианта обеим целям одновременно. В работе [18] в качестве функции принадлежности $\mu(x)$ параметра $x$ поставленной цели $\bar{x}$ в подобной трактовке предлагается использовать зависимость вида

$$
\mu(x)=\frac{1}{1+(x-\bar{x})^{2}} .
$$

Тогда для нашего примера функция принадлежности $\mu_{1}\left(r_{1}\right)$ каждого допустимого варианта Цели 1 определяется выражением

$$
\mu_{1}\left(r_{1}\right)=\frac{1}{1+r_{1}^{2}}
$$

а функция принадлежности $\mu_{2}\left(r_{2}\right)$ каждого допустимого варианта Цели 2 - выражением

$$
\mu_{2}\left(r_{2}\right)=\frac{1}{1+r_{2}^{2}}
$$

Функция принадлежности допустимого варианта обеим поставленным целям одновременно $\mu$ определяется согласно работе [18] как наименьшее среди значений функций принадлежности $\mu_{1}$ и $\mu_{2}$ :

$$
\mu=\min \left(\mu_{1}, \mu_{2}\right)
$$


Поскольку значение $\mu$, рассчитанное для каждого варианта (рис. 2), характеризует степень принадлежности этого варианта обеим поставленным целям одновременно, то из двух рассматриваемых вариантов назначения напусков следует выбрать тот, у которого наибольшая степень принадлежности, чему соответствует максимальное значение $\mu$.

В табл. 1 приведены результаты расчетов значений функции $\mu$ для допустимых вариантов поковок, изображенных на рис. 2, в соответствии с которыми и приведенными выше рассуждениями интерсубъективным вариантом допустимой поковки, наиболее удовлетворяющей сформулированным целям, следует считать вариант 2.

Следует отметить, что решение поставленной задачи, отраженное в табл. 1, не учитывает важности Целей 1 и 2. Естественно при большом объеме заказа и высокой стоимости металла более важной является Цель 1 «экономия металла» и наоборот.

Таблица 1 - Результаты расчетов значений $\mu$

\begin{tabular}{|c|c|c|}
\hline Характеристики & Вариант 1 & Вариант 2 \\
\hline$M$, кг & 10550 & 12630 \\
\hline$M_{5}$, кг & \multicolumn{2}{|c|}{10550} \\
\hline$M_{\Gamma}$, Кг & 0 & 0,29 \\
\hline$r_{1}=\frac{M_{5}-M_{5}}{M_{\Gamma}-M_{5}}$ & 1 & 0,92 \\
\hline$\mu_{1}\left(r_{1}\right)=\frac{1}{1+r_{1}^{2}}$ & 1 & 0,75 \\
\hline$r_{2}=\frac{V^{C}}{V_{5}^{C}}$ & 0,5 & 0,64 \\
\hline$\mu_{2}\left(r_{2}\right)=\frac{1}{1+r_{2}^{2}}$ & 0,5 & 0,64 \\
\hline$\mu=\min \left(\mu_{1}, \mu_{2}\right)$ & & \\
\hline
\end{tabular}

Для учета относительной важности сформулированных целей в работе [18] предлагается ввести дополнительные параметры:

$$
\alpha_{1} \in[0,1], \alpha_{2} \in[0,1], \alpha_{1}+\alpha_{2}=1,
$$

где $\alpha_{1}, \alpha_{2}$ - относительные важности Целей 1 и 2 , и результирующую функцию принадлежности $\mu$ определять в виде

$$
\mu=\min \left(\mu_{1}^{\alpha_{1}}, \mu_{2}^{\alpha_{2}}\right) .
$$

Для иллюстрации влияния важностей поставленных целей на решение задачи предположим, например, что для пользователя первостепенное значение имеет Цель 1 «экономия металла», а Цель 2 «снижение трудоемкости» значения не имеет, т.е. $\alpha_{1}=1, \alpha_{2}=0$. Результаты расчетов приведены в табл. 2 . 
Таблица 2 - Результаты расчета значений $\mu$ при целях различной важности

\begin{tabular}{|c|c|c|}
\hline Характеристики & Вариант 1 & Вариант 2 \\
\hline$\mu_{1}\left(r_{1}\right)=\frac{1}{1+r_{1}^{2}}$ & 1 & 0,92 \\
\hline$\mu_{1}\left(r_{1}\right)^{1}$ & 1 & 0,92 \\
\hline$\mu_{2}\left(r_{2}\right)=\frac{1}{1+r_{2}^{2}}$ & 0,5 & 0,64 \\
\hline$\mu_{2}\left(r_{2}\right)^{0}$ & 1 & 1 \\
\hline$\mu=\min \left(\mu_{1}, \mu_{2}\right)$ & 1 & 0,92 \\
\hline
\end{tabular}

В этом случае интерсубъективным вариантом допустимой поковки, наиболее удовлетворяющей сформулированным целям, естественно следует считать вариант 1, представленный на рис 2.

\section{2. Заключение}

Разработан механизм автоматизированного управления пользователем процессом назначения напусков на поковки типа валов при автоматизированном проектировании технологического процесса ковки посредством варьирования относительных важностей поставленных целей. Изменяя относительную важность целей в пределах от 0 до 1 , пользователь имеет возможность проанализировать множество вариантов спроектированного технологического процесса ковки и выбрать подходящий с его точки зрения вариант.

\section{Благодарность}

Работа выполнена по бюджетной теме № 0391-2016-0004 (2/p AAAA-A18118020790142-9).

\section{Список литературы}

1. Karayel D., Ozkan S.S. Distributed multi-agent system approach for sheet metal forming // Journal of Materials Processing Technology. - 2006. - Vol. 177, no. 1-3. - P. 327-330. DOI: 10.1016/j.jmatprotec.2006.04.039.

2. Zhang J., Chen Q. Characteristic finite strip analysis of sheet materials on multiintelligence agents // Advanced Materials Research. - 2013. - Vol. 662. - P. 657-660. DOI: $10.4028 / w w w . s c i e n t i f i c . n e t / A M R .662 .657$.

3. Каверин A. ADEM - современная технология подготовки кузнечного производства // САПР и графика. - 2005. - № 3. - С. 1.

4. Разработка и применение программного обеспечения для автоматизированного проектирования и моделирования процессов ковки и горячей штамповки / В. С. Чесноков, Б. Г. Каплунов, Н. Е. Возмищев и др. // Кузнечно-штамповочное производство. Обработка материалов давлением. - 2008. - № 9. - С. 36-44.

5. Construction of PC-based intelligent CAD system for cold forging process design - integration of CAD system and development of input method / S. Sakamoto, T. Katayama, R. Yokogawa et al. // Journal of Materials Processing Technology. - 2001. - Vol. 119, no. 1-3. - P. 58-64. 
6. Development of a CAD/CAM system for the closed-die forging process / M. Jolgaf, A. M. S. Hamouda, S. Sulaiman et al. // Journal of Materials Processing Technology. - 2003. Vol. 138, nos. 1-3. - P. 436-442. - DOI 10.1108/02644400910943635.

7. Development of integrated and intelligent design and analysis system for forging processes / D. Y. Yang, Y. T. Im, Y. C. Yoo et al. // CIRP Annals - Manufacturing Technology. - 2000. Vol. 49, no. 1. - P. 177-180.

8. Система управления качеством проектирования технологических процессов ковки / В. Н. Трубин, В. И. Макаров, С. Н. Орлов и др. - М. : Машиностроение, 1984. - 184 с.

9. Канюков С. И., Арзамасцев С. В. Система автоматизированного проектирования технологии ковки ступенчатых валов // Кузнечно-штамповочное производство. Обработка материалов давлением. - 1995. - № 9. - С. 13-14.

10. Интеллектуальная САПР технологических процессов ковки валов на молотах / А. В. Коновалов, С. В. Арзамасцев, С. Д. Шалягин и др. // Заготовительные производства в машиностроении. - 2010. - № 1. - С. 20-23.

11. Коновалов А. В., Гагарин П. Ю., Шалягин С. Д. Объектная модель ковки коротких поковок // Автоматизация и современные технологии. - 2010. - № 6. - С. 11-15.

12. ГОСТ 7062-90. Поковки из углеродистой и легированной стали, изготовляемые ковкой на прессах. Припуски и допуски.

13. Люгер Д. Ф. Искусственный интеллект: стратегии и методы решения сложных проблем / пер. с англ. - 4-е изд.- М. : Издательский дом «Вильямс», 2003. - 864 с.

14. Стюарт Р., Норвиг П. Искусственный интеллект: современный подход. - 2-е изд. М. : Издательский дом «Вильямс», 2006. - 1408 с.

15. Боргест Н. М. Онтология проектирования: теоретические основы: учебное пособие. Ч. 1 : Понятия и принципы. - Самара : Изд-во СГАУ, 2010. - 92 с.

16. Виттих В. А. Введение в теорию интерсубъективного управления. - Самара : Самарский научный центр РАН, 2013. - 60 с.

17. Рыжов А. П. Элементы теории нечетких множеств и ее приложений. - М. : ДиалогМГУ, 1998. - $81 \mathrm{c.}$

18. Беллман Р., Заде Л. Принятие решений в расплывчатых условиях. Вопросы анализа и процедуры принятия решений. - М. : Мир, 1976. - С. 172-215. 